\title{
DArT markers: diversity analyses and mapping in Sorghum bicolor Emma S Mace ${ }^{\dagger 1}$, Ling Xia ${ }^{\dagger 2}$, David R Jordan1, Kirsten Halloran ${ }^{1}$, Dipal K Parh ${ }^{3,4}$, Eric Huttner ${ }^{2}$, Peter Wenzl ${ }^{2}$ and Andrzej Kilian*2
}

\author{
Address: ${ }^{1}$ The Department of Primary Industries \& Fisheries, Queensland (DPI\&F), Hermitage Research Station, Warwick, QLD 4370, Australia \\ ${ }^{2}$ Diversity Arrays Technology P/L, PO Box 7141, Yarralumla ACT 2600, Australia, ${ }^{3}$ School of Land and Food Sciences, University of Queensland, \\ Brisbane, QLD 4072, Australia and ${ }^{4}$ Bureau of Sugar Experiment Station, DNRP, 50 Meiers Road, Indooroopilly, QLD 4068, Australia \\ Email: Emma S Mace - emma.mace@dpi.qld.gov.au; Ling Xia - ling@diversityarrays.com; David R Jordan - david.r.jordan@dpi.qld.gov.au; \\ Kirsten Halloran - kirsten.halloran@dpi.qld.gov.au; Dipal K Parh - d.parh@bses.org.au; Eric Huttner - e.huttner@diversityarrays.com; \\ Peter Wenzl - p.wenzl@diversityarrays.com; Andrzej Kilian* - a.kilian@diversityarrays.com \\ * Corresponding author †Equal contributors
}

Published: 22 January 2008

BMC Genomics 2008, 9:26 doi:10.1/86/147|-2164-9-26
Received: 28 June 2007

Accepted: 22 January 2008

This article is available from: http://www.biomedcentral.com/I47I-2/64/9/26

(C) 2008 Mace et al; licensee BioMed Central Ltd.

This is an Open Access article distributed under the terms of the Creative Commons Attribution License (http://creativecommons.org/licenses/by/2.0), which permits unrestricted use, distribution, and reproduction in any medium, provided the original work is properly cited.

\begin{abstract}
Background: The sequential nature of gel-based marker systems entails low throughput and high costs per assay. Commonly used marker systems such as SSR and SNP are also dependent on sequence information. These limitations result in high cost per data point and significantly limit the capacity of breeding programs to obtain sufficient return on investment to justify the routine use of marker-assisted breeding for many traits and particularly quantitative traits. Diversity Arrays Technology $\left(\mathrm{DArT}^{\mathrm{TM}}\right)$ is a cost effective hybridisation-based marker technology that offers a high multiplexing level while being independent of sequence information. This technology offers sorghum breeding programs an alternative approach to whole-genome profiling. We report on the development, application, mapping and utility of $\mathrm{DArT}^{\mathrm{TM}}$ markers for sorghum germplasm.
\end{abstract}

Results: A genotyping array was developed representing approximately 12,000 genomic clones using Pstl+Banll complexity with a subset of clones obtained through the suppression subtractive hybridisation (SSH) method. The genotyping array was used to analyse a diverse set of sorghum genotypes and screening a Recombinant Inbred Lines (RIL) mapping population. Over 500 markers detected variation among 90 accessions used in a diversity analysis. Cluster analysis discriminated well between all 90 genotypes. To confirm that the sorghum DArT markers behave in a Mendelian manner, we constructed a genetic linkage map for a cross between R931945-2-2 and IS 8525 integrating DArT and other marker types. In total, 596 markers could be placed on the integrated linkage map, which spanned I43I.6 cM. The genetic linkage map had an average marker density of $\mathrm{I} / 2.39 \mathrm{cM}$, with an average DArT marker density of I/3.9 cM.

Conclusion: We have successfully developed DArT markers for Sorghum bicolor and have demonstrated that DArT provides high quality markers that can be used for diversity analyses and to construct medium-density genetic linkage maps. The high number of DArT markers generated in a single assay not only provides a precise estimate of genetic relationships among genotypes, but also their even distribution over the genome offers real advantages for a range of molecular breeding and genomics applications. 


\section{Background}

Sorghum is a major staple food and fodder crop grown worldwide, with an annual average production of $61 \mathrm{mil}-$ lion tonnes over the past decade [1]. The crop is tolerant of many biotic and abiotic stresses and is often grown in more marginal cropping areas. In developing countries it tends to be a staple food and forage of the poor. In developed countries it is used primarily as an animal feed. Sorghum is often preferentially grown in both situations as it is better adapted to water limited environments than other cereal crops.

Investment in sorghum breeding and genomic resources has been less than for the other major cereals rice, wheat, maize and barley. Interest has focused on the crop due to its drought resistance and small genome size ( 760 Mb) compared to close relatives maize ( $2500 \mathrm{Mb}$ ) and sugarcane (2550 to $4200 \mathrm{Mb}$ ) [2-4]. In recent years the potential of sorghum as a biofuel crop has led to additional investment culminating in the sequencing of the sorghum genome [5].

Numerous studies have demonstrated that sorghum is very diverse crop, with cultivated sorghums exhibiting great phenotypic variability. The cultivated germplasm has been classified into five major races (bicolor, caudatum, durra, guinea and kafir) and 10 intermediate races based on panicle and spikelet morphology [6]. In order to exploit this diversity at the genotypic level, an efficient marker system is required. Many molecular marker technologies have been developed and applied to studying patterns of genetic diversity in sorghum germplasm collections and in breeding programs, including RFLPs [7-9], RAPDs [10,11], ISSRs [12], SSRs [13-19] and AFLPs $[17,20]$. However, the major limitation to the widespread use of current marker technologies in applied sorghum breeding programs and germplasm collections is the high cost per data point. Applications that require whole genome scans such as pedigree analysis [21], association mapping [22] and mapping as you go (MAYG) [23], or large scale genotyping of germplasm collections [24] are not cost effective using current technologies.

The current molecular marker technologies have characteristics which additionally affect the level of genome coverage, their discrimination ability, reproducibility and technical and time demand. A number of the limitations associated with the different marker technologies can be overcome by utilising specialised hardware such as high throughput capillary electrophoresis machines, which can impact on discrimination ability, reproducibility and speed. However, the majority of the limitations are related to the sequential nature and high assay costs of the marker technologies, in addition to reliance on DNA sequence information. Diversity arrays technology (DArT) can over come these limitations and has been developed as a hybridisation-based alternative to the majority of gelbased marker technologies currently in use. The DArT genotyping method was developed originally for rice [25] and has subsequently been applied to many other plant species, including barley [26], cassava [27], Arabidopsis [28], pigeonpea [29] and wheat [30]. DArT has been also applied to a number of animal species and microorganisms [31]. The DArT methodology offers a high multiplexing level, being able to simultaneously type several thousand loci per assay, while being independent of sequence information. DArT assays generate wholegenome fingerprints by scoring the presence versus absence of DNA fragments in genomic representations generated from genomic DNA samples through the process of complexity reduction.

This paper reports the results of a study designed to (1): develop a sorghum diversity array for DArT genotyping, (2): determine linkage map positions of polymorphic DArTs and (3): assess utility of DArT technology in diversity analyses on a set of diverse sorghum lines, including selected lines from the Department of Primary Industries and Fisheries (DPI\&F) sorghum breeding program. In order to evaluate the discriminatory ability of DArTs, efforts have been made to include the same genotypes used in genetic diversity studies based on other molecular marker technologies.

\section{Results \\ Evaluation of complexity reduction methods and array development}

The initial tests of DArT performance in sorghum were done on eight sorghum genotypes (Additional File 1). Based on our positive experience with PstI-based genomic representations [25] we initially evaluated several combinations of PstI with different frequently cutting restriction enzymes (RE) as a complexity reduction approach for sorghum. DNA samples from the eight sorghum genotypes were digested with PstI and several frequently cutting RE (PstI+TaqI，Pst I+MseI，Pst I+ApoI，PstI+AluI，PstI+Ban II, $P s t \mathrm{I}+B s t \mathrm{NI}$ and Pst $+A f l \mathrm{III})$, ligated to a PstI adapter and then amplified with the PstI-0 primer. Gel analysis of the PCR products showed that a uniform smear (without major bands) only appeared in the PstI+BanII combination. Other RE combinations gave a smear with one or more dominant bands. Such strong bands represent highly amplified restriction fragments and correspond to abundant repetitive sequences in the representation; $\mathrm{a}$ feature which is highly detrimental to DArT performance [32].

AFLP-like analysis was performed to estimate the fragment number in the PstI+BanII representation following the approach utilized by Xia et al. [27]. Four primers con- 
taining 3 selective bases at the 3'end were used for the amplification. For all four primers, a large number of fragments were visible on the gel, making a precise estimate of the total number of fragments impossible (data not presented). Interestingly, a similar approach resulted in an easily quantifiable number of bands in cassava which has a similar genome size to sorghum and even in barley, which has genome size almost seven times larger than sorghum. The results suggest that the Pst I+BanII representation in sorghum has a larger number of unique fragments than the Pst $\mathrm{I}+B s t \mathrm{NI}$ representation in barley which was reported to contain 1,546 markers placed on the integrated map of barley genome [26].

Based on the extended PstI+BanII sub-libraries and the additional libraries generated based on the genomic subtraction (SSH) method [33], the best DArT markers from the initial experiments were "cherry picked" and a "rearray library" with 768 polymorphic clones was created. In addition to these 768 polymorphism-enriched clones, a further 5367 clones from Pst I+BanII Library C were used for all genotyping work reported here.

\section{Genetic relationships between sorghum lines revealed by DArT}

The selected DArT clones were tested for their ability to resolve genetic relationships among a set of 90 lines. The reproducibility of the DArT genotyping array was successfully validated by independent assays from the same DNA. The genotypes selected represent a significant proportion of the genetic variation in sorghum with all 5 races represented and 5 intermediate races also represented. In addition, the germplasm set included elite lines from breeding programs some of which had high levels of co-ancestry.

DArTsoft analysis (see Materials and Methods) identified 508 markers polymorphic among 90 genotypes typed on the array. The PIC values of these 508 markers were very high with over $69 \%$ of the markers having a PIC value between 0.4 and 0.5 (Table 1 ). The average PIC was 0.41 , higher than the previous DArT studies in barley $(0.38$ [26]) and comparable with cassava (0.42 [27]). The relationship between the quality of the DArT markers (measured as the \% of total variance which existed between the

Table I: Polymorphism Information content (PIC) values for 508 DArT markers

\begin{tabular}{lrr}
\hline PIC value & \# DArTs & \% DArTs \\
\hline $0.5-0.4$ & 353 & 69.5 \\
$0.4-0.3$ & 82 & 16.1 \\
$0.3-0.2$ & 46 & 9.1 \\
$0.2-0.1$ & 21 & 4.1 \\
$0.1-0$ & 6 & 1.2
\end{tabular}

two clusters: present and absent) and the performance of the DArT markers as determined through call rate and PIC was analysed (Table 2). The PIC values were largely independent of marker quality, with only a small reduction in PIC value observed in the lowest quality class $(0.40$ in the lowest quality class vs. 0.44 and 0.42 for the two higher quality classes). As expected, the average call rate decreased with average $\mathrm{Q}$ value. The markers with the highest Q values (above 90\% of total variance between the clusters) had very high average call rates (98\%), while the markers in the lower quality marker classes had lower average call rates and higher standard deviations.

Cluster analysis based on the DICE dissimilarity index and the unweighted neighbour-joining method was performed on the 508 DArT markers for 90 genotypes (Figure 1). This cluster analysis discriminated well between all 90 genotypes and has a cophenetic correlation value of 0.9308, indicating an excellent fit of the similarity matrix data to the tree topology. Thirteen main clusters were identified which correspond well with race and origin of the genotyped lines. In particular a single predominant race or origin could be identified in 9 out of the 13 clusters. Cluster 1 contained 13 genotypes in total, of which 11 were kafir or kafir-caudatum. Cluster 4 contained 9 genotypes, of which 5 were of race durra. Additionally, two Ethiopian durra types (IS 12555C and B35) grouped together within this cluster with a boot strap value of $100 \%$. Cluster 5 consisted of 6 Chinese genotypes of complex racial background. Cluster 6 contained the wild species, S. propinquum and the weedy subspecies, S. bicolor subsp. verticilliflorum (formerly $S$. arundinaceum). Cluster 9 consisted of 2 caudatum genotypes (IS $12656 \mathrm{C}$ and IS 10302). Cluster 10 consisted of 11 genotypes in total, of which 8 were caudatum or caudatum-derived. Cluster 11 consisted of a tight cluster of restorer (R) lines predominately from Australian breeding programs; R9990066, R999017 and R999003 are all progeny of the line R31945-2-2. Cluster 12 is a looser cluster consisting of 15 lines of which 7 were caudatum or caudatum-derived. Finally cluster 13 consisted of 4 genotypes of which 2 are bicolor or bicolor intermediate and S. bicolor subsp. drummondii which is very similar morphologically to the bicolor race.

Table 2: The relationship between the quality and the performance of the DArT markers

\begin{tabular}{llll}
\hline & $100>\mathrm{Q}>90$ & $90>\mathrm{Q}>80$ & $80>\mathrm{Q}>70$ \\
\hline Number of markers & 37 & 236 & 235 \\
Call Rate & $98.03 \pm 1.69$ & $92.75 \pm 3.5 \mathrm{I}$ & $88.0 \mathrm{I} \pm 4.83$ \\
$\mathrm{PIC}$ & $0.44 \pm 0.08$ & $0.42 \pm 0.09$ & $0.40 \pm 0.1 \mathrm{I}$
\end{tabular}




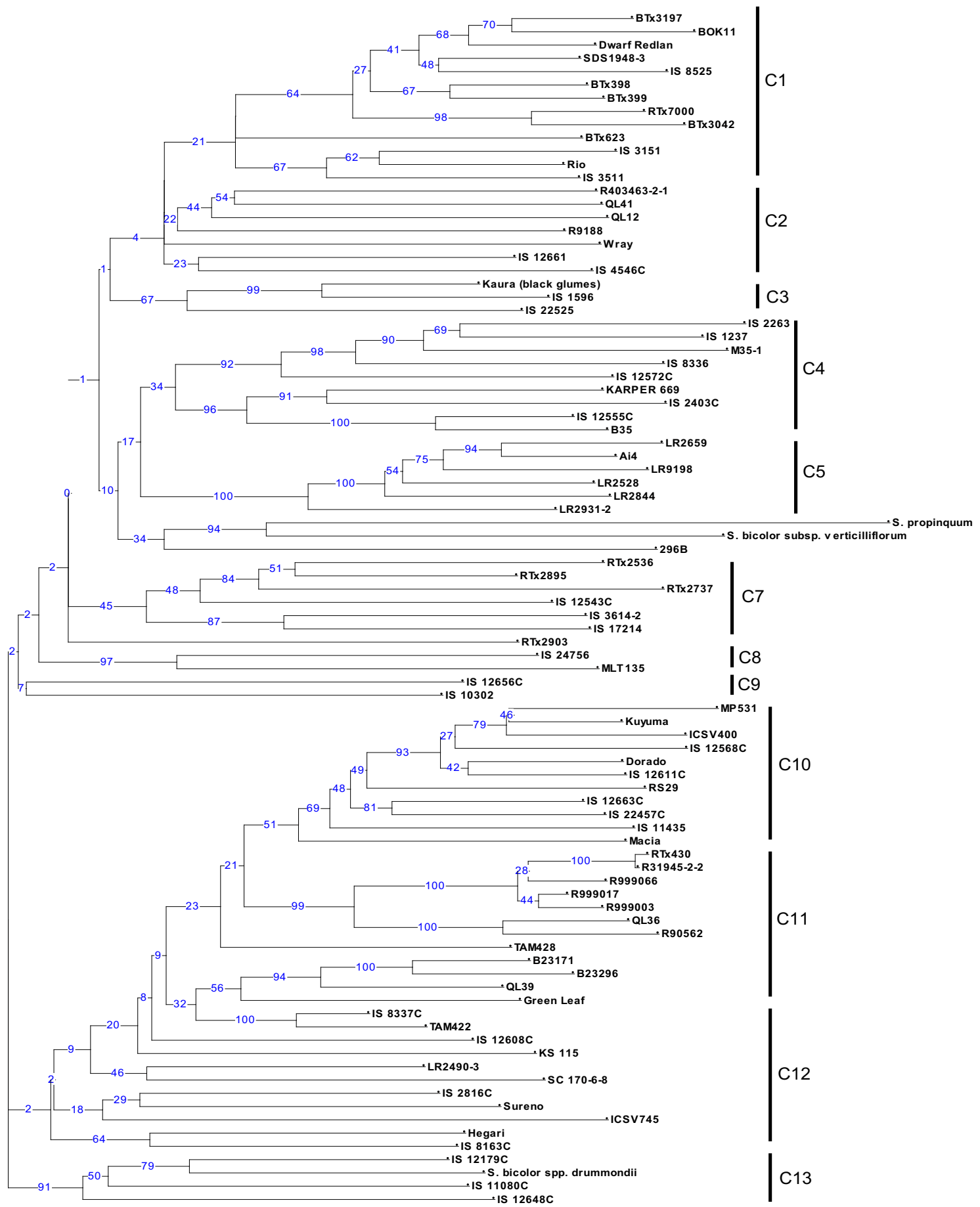

0

Figure I

Neighbor-joining anlaysis of diverse sorghum genotypes based on 508 DArT markers using the DICE similarity coefficient. 13 clusters have been defined. The numbers on the branches indicate bootstrap values (expressed in percentages; based on 100 replications). 


\section{Mapping of DArT loci}

To confirm that the sorghum DArT markers behave in a Mendelian manner, we constructed a genetic linkage map for a cross between R931945-2-2 and IS 8525. We selected 370 DArT markers with Q-values greater than $80 \%$ and merged with the segregation data for 286 markers, consisting of 55 SSRs, 229 AFLPs and 2 morphological markers derived from the original map [34].

In total, 596 markers could be placed on the integrated linkage map, which spanned $1431.6 \mathrm{cM}$ (Table 3 ). The genetic linkage map had an average marker density of $1 /$ $2.39 \mathrm{cM}$, with an average DArT marker density of $1 / 3.9$ cM. The 358 DArT markers included on the map mapped to all 10 chromosomes and are distributed across the genome in a similar way as the 47 SSR and 188 AFLP markers (Figure 2), suggesting that the density of both groups of markers roughly followed the distribution of DNA polymorphism across the genome. The DArT markers accounted for approximately $50 \%$ of the framework markers used in the initial construction of each linkage group, with a high proportion of the DArT markers acting as delegates (Table 3; see M\&M for explanations of framework, delegate and attached markers) indicating a higher level of redundancy compared to the other marker types. We therefore compared the level of redundancy, as determined through co-location, between the DArT markers generated from the 6 subtraction libraries (SSH) versus those developed from the extended PstI+BanII sub-libraries. Of the 358 DArTs included on the map, 172 were SSHdervied and of these 50 (29\%) were redundant, whereas the 186 non-SSH derived DArTs exhibited a reduced level of redundancy $(26 \%)$. Overall redundancy in the map dropped from $33.7 \%$ to $24 \%$ when SSH-derived DArT markers were excluded. Interestingly, the difference in apparent redundancy levels between SSH-derived and "normal" DArT markers was smaller compared to what we observed for tomato and sugarcane (DArT P/L, unpublished) and in fern species Asplenium and moss species Garovaglia (DArT P/L and collaborators, unpublished data).

There was no statistically significant difference between DArT and non-DArT markers in the distribution of parental alleles across the genome. Twelve DArTs were removed during the course of map construction either because of lack of linkage to other markers, or as they formed small linkage groups containing only DArTs which did not link to the known chromosomes.

\section{Discussion \\ Sorghum DArT markers}

This is the first report of the use of DArT technology in sorghum and our results demonstrate that the sorghum DArT markers are high quality, as assessed by their call rate, scoring reproducibility and PIC values. The DArT marker quality parameters measured for the sorghum array are comparable to those obtained for pigeonpea [29], barley [26] and cassava [27] and wheat [30].

\section{Utility of DArTs for diversity analysis}

Among the criteria for genetic markers that are to be used for fingerprinting and marker-assisted selection is a high level of polymorphism [15]. Clearly, sorghum DArTs meet this criterion, with over 69\% of the 508 DArTs revealing polymorphism between the set of 90 sorghum lines having a PIC value between $0.5-0.4$; 0.5 being the highest PIC value expected for a bi-allelic marker system.

Table 3: Summary of the genetic linkage map based on a cross between R3 I 945-2-2 and IS 8525. The genetic linkage map was constructed using DArTs, AFLPs and SSRs. The total length of each chromosome, the total number of markers, the total number of DArTs and the number of framework, delegate and attached markers per chromosome are detailed. For the last three columns, the number of DArTs in each class is given in parentheses

\begin{tabular}{|c|c|c|c|c|c|c|}
\hline SBI & Length (cM) & Total \# markers & \# DArTs & \# framework* & \# delegate* & \# attached* \\
\hline I & 188.1 & 94 & 65 & $31(16)$ & $25(24)$ & $38(25)$ \\
\hline 2 & 135.6 & 48 & 31 & $31(19)$ & $9(9)$ & $9(3)$ \\
\hline 3 & 83 & 31 & 19 & $21(I I)$ & $4(4)$ & $6(4)$ \\
\hline 4 & 133.9 & 70 & 49 & $28(16)$ & $18(18)$ & $24(15)$ \\
\hline 5 & 130.4 & 81 & 43 & $38(17)$ & $18(13)$ & $25(13)$ \\
\hline 6 & |57.| & 61 & 23 & $36(13)$ & $9(5)$ & $16(5)$ \\
\hline 7 & 120.5 & 40 & 24 & $26(15)$ & $5(5)$ & $9(4)$ \\
\hline 8 & 184.5 & 75 & 51 & $41(26)$ & $14(11)$ & $20(14)$ \\
\hline 9 & | 49.3 & 40 & 19 & $24(7)^{\prime}$ & $9(7)$ & $7(5)$ \\
\hline 10 & 149.2 & 56 & 34 & $26(9)$ & $6(5)$ & $25(20)$ \\
\hline Totals & 1431.6 & 596 & 358 & 302 (149) & 117 (101) & $179(108)$ \\
\hline
\end{tabular}

\footnotetext{
* 'Framework' markers are defined as those that could be ordered with a jack-knife value of $90 \%$ or greater using the MultiPoint software; Delegate markers are defined as those that map to the same location as the representative framework marker for a specific locus; Attached markers are those that initially were excluded from the framework map as they caused unstable neighborhoods but were included in the final, complete map by assigning them to the best intervals on the framework map.
} 
SBI-01

SBI-02

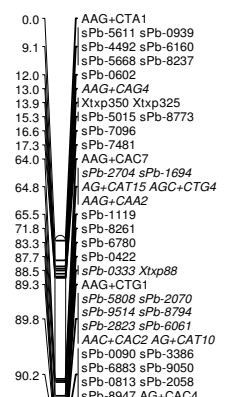

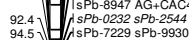

$96.1)=\left(\begin{array}{ll}95 \mathrm{sP}-0274 \\ \mathrm{AAC}+\mathrm{CAC} 1\end{array}\right.$

$\left.\begin{array}{l}112.2 \\ 124.5\end{array}\right)\left(\begin{array}{l}\text { Xtxp37 SPD-3930 } \\ \mathrm{sPb}-0509 \\ \mathrm{ACC}+\mathrm{CTC1}\end{array}\right.$

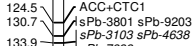

$133.9-1,1=0$

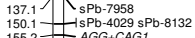

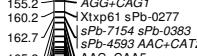

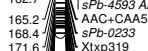

174.1
176.5

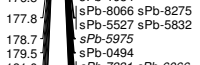

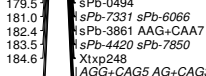

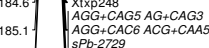

$\left.\begin{array}{l}185.5 \\ 188.1\end{array}\right] \quad \begin{aligned} & S P b-2729 \\ & \text { Xtxp316 } \\ & \text { ACA+CAA2 }\end{aligned}$

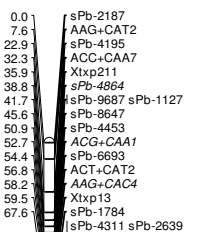

68.9 sPb-6700 sPb-10

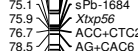

78.5. 珹 $\begin{aligned} & \mathrm{ACC}+\mathrm{CAC6} \\ & \mathrm{SPb}-0073 \\ & \mathrm{SPh}-7702\end{aligned}$

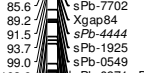

3.6 $=4 \mathrm{sPb}-0971 \mathrm{sPb}-951$

10.3. $\begin{gathered}\mathrm{AAG}+\mathrm{CAC6} \\ \mathrm{sPb}-6663 \mathrm{Xtxp} 31\end{gathered}$

1.5
$\mathrm{sPb}-4054$
$s P b-7153$

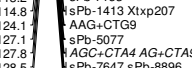

$\left.\begin{array}{l}127.8 \\ 128.5 \\ 135.6\end{array}\right]\left[\begin{array}{l}\mathrm{SPb}-5077 \\ \text { A AGC+CTA4 AG+CCAS } \\ \text { isPb-7647 spb-8896 } \\ \mathrm{sPb}-7015\end{array}\right.$

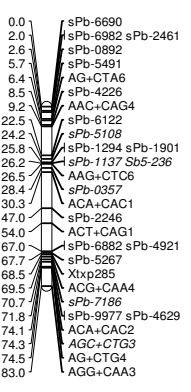

SBI-03

SBI-04

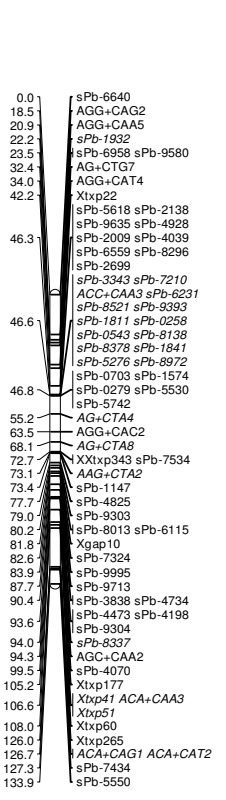

SBI-05

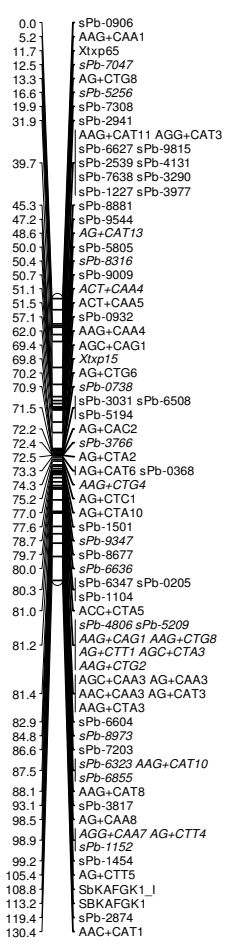

SBI-10
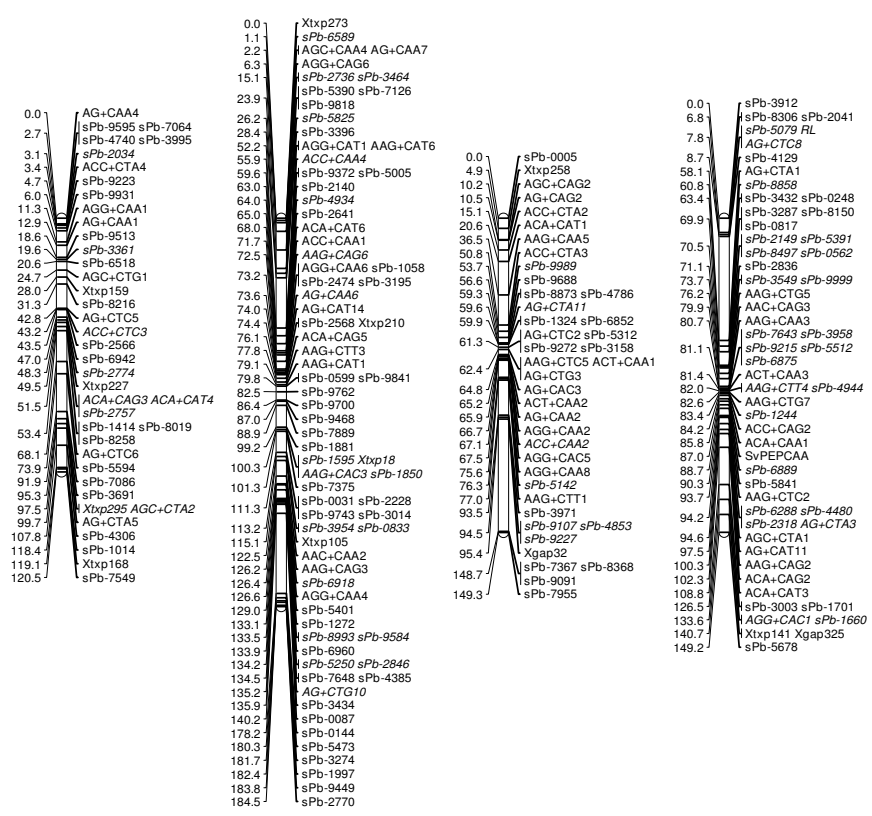

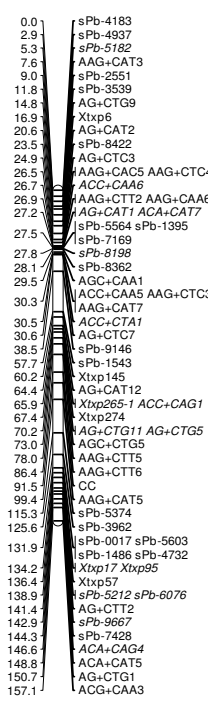

Figure 2

Genetic linkage map for a cross between R3 I 945-2-2 and IS 8525. Genetic distances are expressed as cumulative map distances from position 0.0 (first locus of $L G$ ) in $\mathrm{CM}$ (Kosambi estimates). Locus names in bold indicate framework markers; locus names in italics indicate attached and delegate markers. 
Additionally, this study meets all the criteria proposed for evaluating the use of molecular markers for large scale germplasm diversity analyses [35]; a large number of markers, thorough representation of these markers on a genetic linkage map of the species and the selection of monolocus probes. The 508 DArT markers permitted the unique identification of all 90 genotypes including closely related backcross derived lines. The cluster analysis revealed that the 90 genotypes examined showed a clear demarcation of the germplasm according to their racial classification, consistent with previous studies using RFLPs [9] and SSRs and AFLPs [17]. The bicolor race was found to be highly variable in this study, shown by its presence in multiple clusters, as also noted by previous studies $[8,9,14]$. In fact, it has been noted previously that race bicolor resembles spontaneous weedy sorghums and is thought to be the race most closely related to wild sorghums [18] and also the most primitive grain sorghum [10]. Indeed, the bicolor race accession IS 12179C included in this study, groups together with the weedy sorghum, S. bicolor subsp. drummondii, in cluster 13 (Figure 1).

The race caudatum is one of the most important agronomically, providing genes for high yield and excellent seed quality. It has become one of the most important sources of germplasm in modern breeding programs throughout the world and in this study, the caudatum race genotypes were clearly demarcated in clusters 9, 10 and 12. In contrast, a previous study [18] found no significant differences in diversity between the races, except the kafir race which was less diverse. Low genetic diversity was also observed in the kafir race in this study, with all the kafir race accessions constituting a specific cluster (cluster 1). These results are in agreement with the recent origin and restricted geographic distribution of this race, together with recent studies using SSRs [14] and RFLPs [9].

In addition to the observed clustering on racial groups, differences between the genotypes based on their status as $\mathrm{B}$ (maintainer female) and $\mathrm{R}$ (male parental restorer) lines was also noted. In this study, there was less variation among the B-lines than the R-lines, with the B-lines clustering tightly together in only 2 of the 13 clusters, whereas the R-lines grouped more loosely in 5 clusters. It has also been noted [17] that the lower levels of diversity among elite $\mathrm{B}$-lines vs. R-lines is expected since B-lines are required to produce high quality male-sterile A-lines. The development of new A/B-lines is more difficult compared to R-line development and hence B-line development is more restrictive and slower to incorporate new germplasm. Where pedigree data was available, groupings associated with these pedigree relationships were observed.
Comparisons of the discrimination ability for DArT markers and their ability to reflect pedigree backgrounds in contrast to other marker types is made much simpler when over-lapping sets of germplasm are used in a number of studies. Seventeen genotypes included in this study were examined by Menz et al. [17], 54 genotypes were in common with Ritter et al. [20] and 11 with Tao et al. [7]. Very similar groupings of genotypes were observed, e.g. the over-lapping genotypes in cluster 1 also group together in previous studies $[17,20]$, e.g. BTx3197 and BOK11 grouping tightly together in Menz et al. [17], in addition to RTx7000 and Btx3042. Similarly, ICSV400, MP531 and Macia group together based on AFLPs $[17,20]$ and DArTs (cluster 10), and QL41 and R9188 group together based on RFLPs [7] and DArTs (cluster 2).

The separation of "wild" sorghums from cultivated germplasm seems to be less pronounced compared to the results based on SSR data [18]. This is not surprising, as the array used for genotyping all materials in this study was developed using mostly DNA from cultivated materials. Even though three accessions from wild relatives were included in library construction, their "private" alleles were highly "diluted" by common alleles and those which were "private" to the cultivated material. The design of the array did not substantially change the topology of trees generated from DArT data, but would reduce the distance between the wild and cultivated samples. We expect that the distance reduction would be up to two-fold, as we were effectively finding unique " 0 " scores for the wild materials, but less effectively unique " 1 " scores. Such ascertainment bias is not limited to DArT, but is a feature of many marker systems. In fact the ascertainment bias will be significantly larger for SNP technologies, as most SNP markers are developed from a small number of samples/chromosomes in a limited number of populations, even in well resourced human studies $[36,37]$. The effect of such "marker source sampling bias" was recognised in many human studies, e.g. on population migration rate [38], population mutation and recombination rate estimates [39] and on Linkage Disequilibrium (LD) estimates [40]. Marker panels in DArT are developed using large and representative sampling from target populations [32] and can be easily expanded by cloning libraries from the germplasm pools for which precise relatedness estimates would be required.

\section{Distribution of DArT loci in the sorghum genome}

The integrated genetic linkage map comprising DArTs, AFLPs and SSRs clearly demonstrates that the new sorghum DArT markers behave in a Mendelian manner. In total, 358 DArTs were mapped to 257 unique loci. The higher level of redundancy of the DArT markers is reflected by the higher number of AFLP and SSR markers having unique segregation patterns. However, the markers 
on the DArT array used in this study were not filtered for redundancy, whereas the SSR/AFLP data set had previously undergone curation [34], to remove markers with redundant segregation patterns. Also, the total number of DArT markers was higher than in other marker classes, therefore the apparent redundancy would need to be also corrected for sample size. After applying such sample-size correction to STMP markers in the linkage map of a cross between two wheat cultivars Cranbrook and Halberd, a lower level of redundancy was found for the DArT markers [30].

The total length of the integrated genetic linkage map was $1431.6 \mathrm{cM}$, with an average DArT marker density of 1 per every $3.9 \mathrm{cM}$. The total map length is comparable to other recently reported sorghum genetic maps, being slightly shorter than the $1713 \mathrm{cM}$ high-density genetic linkage map based on 2926 AFLP, RFLP and SSR markers [41], and slightly longer than the $1059.2 \mathrm{cM}$ genetic linkage map based on 2050 RFLP probes [42]. Although the DArT markers are distributed across the genome in a similar way to the non-DArT markers, there are genomic regions containing significant excesses or paucity of markers, e.g. the centromeric region of SBI-05 has a higher than average marker density of $1 / 0.64 \mathrm{cM}$ and the distal ends of SBI-01 and SBI-10 have marker-poor regions with gaps spanning over $30 \mathrm{cM}$. These areas of low marker density may correspond to regions of similar ancestry or identity by descent (IBD) in the germplasm included in the initial diversity representation. In addition, lines with photoperiod sensitivity and tall stature were under represented in the diversity set used to develop the DArT markers. These regions of low marker density may be therefore associated with genomic regions that were identical by descent or that had very limited genetic variability in the initial diversity representation. The marker-dense regions appear to correspond to the centromeric regions, a feature that has been observed previously [42]. This is also supported by the recent observation that the pericentromeric heterochromatic regions of sorghum chromosomes show much lower rates of recombination ( $8.7 \mathrm{Mbp} / \mathrm{cM})$ compared to euchromatic regions $(\sim 0.25 \mathrm{Mbp} / \mathrm{cM})$, with the average rate of recombination across the heterochromatic portion of the sorghum genome being 34 -fold lower than recombination in the euchromatic region [43]. It should however be noted that clustering around the centromeres is observed for both DArT and non-DArT markers, due to the centromeric suppression of recombination. Interestingly, DArT markers were significantly less clustered at most centromeric regions of barley chromosomes compared to non-DArT markers on the integrated map containing approximately 3,000 markers [26]. We will be in position to rigorously test if this difference in marker position holds true in sorghum only after completion of building the consensus map integrating approximately
1,000 DArT markers with similar number of other types of markers [Mace et al in preparation].

\section{Conclusion}

We have successfully developed DArT markers for Sorghum bicolor and have demonstrated that DArT provides high quality markers that can be used for diversity analyses and to construct medium-density genetic linkage maps. The high number of DArT markers generated in a single assay not only provides a precise estimate of genetic relationships among genotypes, but also their even distribution over the genome offers real advantages for a range of molecular breeding and genomics applications. Additionally, the availability of the sorghum whole genome sequence by the end of 2007 offers very exciting opportunities for assessing the colinearity of the DArT markers on the genetic linkage maps with the markers on the sequence map. As DArT assays are performed on highly parallel and automated platforms the cost of datapoint (a few cents per marker assay) is reduced by at least an order of magnitude compared to current, gel-based technologies.

\section{Methods \\ Source of DNA}

The sorghum accessions used to prepare DArT libraries represent the genetic diversity present in the cultivated species (S. bicolor subsp. bicolor) with all 5 races and 5 intermediate races represented, two weedy subspecies ( $S$. bicolor subsp. drummondii and subsp. verticilliflorum) and a wild species, $S$. propinqum (Additional File 1). In addition, the germplasm set included elite lines from breeding programs some of which had high levels of co-ancestry. DNA was extracted using a modified CTAB-based extraction protocol $[44,45]$.

\section{Development of DArT for sorghum}

Several DArT arrays were built in the course of this study. For each of these arrays, a genomic representation was generated from a mixture of sorghum lines using the PstIbased complexity reduction method previously described [26]. Libraries were prepared as described previously [25]. Two extended PstI+BanII sub-libraries were subsequently generated using DNA from 31 and 94 genotypes, respectively (Additional File 2). In addition, six libraries were generated by applying the SSH method to genomic representations [33]. Drivers and testers used in the subtraction libraries construction were as shown in Table 4. DNA of drivers and testers was digested by PstI/Bst NI and ligated to the PstI adaptor. The digestion/ligation products were amplified using the PstI-0 primer. The resulting PCR products were digested with a RE mixture containing DpnII, HpyCH4IV, MseI and NlaIII. Subtraction was done in a 30:1 ratio of driver to tester and carried out in one and two rounds of subtractive hybridization. After final amplifica- 
tion, the subtraction products were cloned as detailed previously [27]. The sorghum libraries utilized for the initial marker discovery are summarized in the supplementary material (Additional File 3). Clones from all libraries with the exception of Pst I+BanII Library C were used to create arrays in order to genotype several hundred sorghum accessions (data not presented). The best markers from the initial experiments were "cherry picked" to assemble the "re-array library" with 768 polymorphic clones. Clones from this library together with 5367 clones from PstI+BanII Library C were used for all genotyping work reported here. The re-array library was created using the PstI+BanII and the subtraction (SSH) libraries. Details of the re-array library and other libraries used for sorghum genotyping are included in the supplementary material (Additional Files 2 \&3).

\section{DArT genotyping}

Genotyping was performed essentially as described in references 26 and 30. Briefly, each genomic DNA sample is subjected to the PstI+BanII complexity reduction method. The resulting genomic representation is labelled with fluorescent nucleotides and hybridised on a microarray printed with the DArT clones. A typical experiment is performed on about 94 samples of genomic DNA. Following hybridisation and washing, the microarrays for an experiment are scanned, the images are analysed and the score of each marker is calculated for each sample by dedicated software DArTsoft: markers are scored 1 for presence, 0 for absence and X for inability to score. The quality parameter $\mathrm{Q}$ for each marker is calculated by dividing the variance of the hybridisation level for the marker between the 2 clusters (present and absent) by the total variance of hybridisation level of the marker, in the experiment.

\section{Diversity analysis}

A group of 90 sorghum lines (subset detailed in Additional File 1) were genotyped on the re-array library as described previously [30]. The sorghum lines were chosen to provide a reasonable representation of sorghum genetic diversity as well as including elite inbred lines from the DPI\&F and other sorghum breeding programs. Some of the elite lines were quite closely related and were included to demonstrate the discrimination possible with DArT.
The marker scores were subjected to cluster and principal coordinate analysis using the DARwin [46] to visualize the genetic relationships among the lines. Additionally, the polymorphism information content (PIC) of each DArT marker was determined as follows; PIC $=1-\Sigma \mathrm{P}_{\mathrm{i}}{ }^{2}$, where $P_{i}$ is the frequency of the $i$ th allele in the examined genotypes [47].

\section{Genetic mapping}

The lines R931945-2-2, a commercially accepted restorer line in Australia and IS 8525, an Ethiopian line (kafir race), in addition to 92 lines of a $F_{5}$ recombinant inbred line (RIL) population derived from a cross between the two lines were typed using the genotyping array. Clones with $\mathrm{Q}>80 \%$ and a call rate of at least $80 \%$ were selected for mapping. DArTs markers were merged with an existing mapping data set consisting of 286 markers including 55 SSRs and 229 AFLPs [34]. A genetic linkage map was constructed using MultiPoint software [48]. The RIL_Selfing population setting was selected and a maximum threshold $\mathrm{rf}_{\mathrm{s}}$ value of 0.40 was used to initially group the markers into ten linkage groups. Multipoint linkage analysis of loci within each LG was then performed and marker order was further verified through re-sampling for quality control via jack-knifing [49]. Markers that could be ordered with a jack-knife value of $90 \%$ or greater were included as 'framework' markers, with any remaining markers causing unstable neighborhoods being initially excluded from the map, including redundant markers mapping to the same location. Following a repeated multipoint linkage analysis with the reduced set of markers for each LG to achieve a stabilised neighbourhood, the previously excluded markers were attached by assigning them to the best intervals on the framework map and labelled as attached markers. The redundant markers were also included on the final, complete map but labelled as delegates. Finally, the linkage groups were assigned to sorghum chromosomes, SBI-01 to SBI-10 according to recent nomenclature [50]. The Kosambi [51] mapping function was used to calculate the centimorgan $(\mathrm{cM})$ values. The graphical representation of the map was drawn using MapChart software [52].

\section{Authors' contributions}

ESM carried out the diversity and mapping analyses and drafted the manuscript. LX co-developed the DArT tech-

Table 4: Drivers and testers used in the subtraction libraries

\begin{tabular}{lllllll}
\hline & Library I & Library 2 & Library 3 & Library 4 & Library 5 & Library 6 \\
\hline $\begin{array}{llllll}\text { Subtraction method } \\
\text { Library }\end{array}$ & One round & One round & One round & One round & One round & Two rounds \\
Driver & Subtraction-I & Subtraction-2 & Subtraction-3 & Subtraction-4 & Subtraction-5 & Subtraction-6 \\
tester & ISCV745 & ISCV745 & 90562 & IS8525 & $31945-2-2$ & Mixture of drivers \\
& Tester mixture* & 90562 & ISCV745 & $31945-2-2$ & IS8525 & Tester mixture* \\
\hline
\end{tabular}

* The tester mixture was comprised of 90 sorghum genotypes without the four parental genotypes (ICSV745, B90562, R31945-2-2 and IS 8525). 
nology for sorghum. DRJ conceived of the study, and participated in its design and coordination, germplasm development and selection and helped to draft the manuscript. KH was involved in the optimisation of the methodology and participated in the mapping analysis. DKP participated in the mapping analysis. EH participated in the development of the DArT technology and editing of the manuscript. PW contributed to ongoing improvements of the DArT technology and the quality assessment of sorghum clones. AK supervised development of the DArT technology, participated in the study's design and coordination and helped to draft the manuscript.

\section{Additional material}

\section{Additional File 1}

List of sorghum genotypes used for the development of the sorghum DArT array and the diversity analyses. The table includes details of genotype IDs and aliases, race and origin and inclusion status in both the methodology developmental stages and diversity analyses.

Click here for file

[http://www.biomedcentral.com/content/supplementary/14712164-9-26-S1.doc]

\section{Additional File 2}

Summary of sorghum libraries. The table includes details of the number of genotypes used in the development of each sorghum library and the number of clones identified.

Click here for file

[http://www.biomedcentral.com/content/supplementary/14712164-9-26-S2.doc]

\section{Additional File 3}

Libraries used for generation of the genotyping array. The table details the barcode for each sorghum library.

Click here for file

[http://www.biomedcentral.com/content/supplementary/14712164-9-26-S3.doc]

\section{Acknowledgements}

We thank the Australian Grains Research and Development Cooperation [53] for financial support. We thank Robert Henzell, Bert Collard, Mandy Christopher, Sally Dillon and colleagues at Diversity Arrays Technology P/ $L$ for helpful discussions and comments on the manuscript.

\section{References}

I. FAOSTAT [http://faostat.fao.org/default.aspx]

2. Pereira MG, Lee M, Bramel-Cox P, Woodman W, Doebley J, Whitkus $R$ : Construction of an RFLP map in sorghum and comparative mapping in maize. Genome 1994, 37:236-243.

3. Grivet L, D'Hont A, Dufour P, Hamon P, Roqes D, Glaszmann JC: Comparative genome mapping of sugar cane with other species within the Andropogoneae tribe. Heredity 1994, 73:500-508.

4. Devos KM: Updating the 'Crop Circle'. Curr Opin Plant Biol 2005, 8:155-162.

5. Bowers JE, Rokhsar DS, Paterson AH: Update on the sorghum (Sorghum bicolor) genome sequence [abstract]. Plant \& Animal Genomes XV Conference: 13-17 January 2007. San Diego [http:/l www.intl-pag.org].
6. Harlan JR, De Wet JMJ: A simplified classification of cultivated sorghum. Crop Sci 1972, 12:127-176.

7. Tao Y, manners JM, Ludlow MM, Henzell RG: DNA polymorphisms in grain sorghum (Sorghum bicolor (L.) Moench). Theor Appl Genet 1993, 86:679-688.

8. Deu M, Gonzalez-de-Leon D, Glaszmann J-C, Degremont I, Chantereau J, Lanaud C, Hamon P: RFLP diversity in cultivated sorghum in relation to racial differentiation. Theor Appl Genet 1994, 88:838-844.

9. Deu M, Rattunde F, Chantereau J: A global view of genetic diversity in cultivated sorghums using a core collection. Genome 2006, 49: 168-180.

10. Dahlberg JA, Zhang X, Hart GE, Mullet JE: Comparative assessment of variation among sorghum germplasm accessions using seed morphology and RAPD measurements. Crop Sci 2002, 42:29I-296.

II. Ayana A, Bryngelsson T, Bekele E: Genetic variation of Ethiopian and Eritrean sorghum (Sorghum bicolor (L.) Moench) germplasm by random amplified polymorphic DNA (RAPD). Genet Resour Crop Evol 2000, 47:47 I-482.

12. Yang W, de Oliveira AC, Godwin I, Schertz K, Bennetzen JL: Comparison of DNA marker technologies in characterising plant genome diversity: Variability in Chinese sorghums. Crop Sci 1996, 36:1669-1676.

13. Djè Y, Forciolo D, Ater $M$, Lefèbvre $C$, Vekemans $X$ : Assessing population genetic structure of sorghum landraces from Northwestern Morocco using allozyme and microsatellite markers. Theor Appl Genet 1999, 99:157-163.

14. Djè $Y$, Heuertz $M$, Lefèbvre $C$, Vekemans $X$ : Assessment of genetic diversity within and among germplasm accessions in cultivated sorghum using microsatellite markers. Theor Appl Genet 2000, 100:918-925.

15. Smith JSC, Kresovich S, Hopkins MS, Mitchell SE, Dean RE, Woodman WL, Lee M, Porter K: Genetic diversity among elite sorghum inbred lines assessed with simple sequence repeats. Crop Sci 2000, 40:226-232.

16. Kong L, Dong J, Hart GE: Characteristics, linkage-map positions, and allelic differentiation of Sorghum bicolor (L.) Moench DNA simple-sequence repeats (SSRs). Theor Appl Genet 2000, 101:438-448.

17. Menz MA, Klein RR, Unruh NC, Rooney WL, Klein PE, Mullet JE: Genetic diversity of public inbreds of sorghum determined by mapped AFLP and SSR markers. Crop Sci 2004, 44: $1236-1244$.

18. Casa AM, Mitchell SE, Hamblin MT, Sun H, Bowers JE, Paterson AH, Aquadro CF, Kresovich S: Diversity and selection in sorghum: simultaneous analyses using simple sequence repeats. Theor Appl Genet 2005, I I I:23-30.

19. Folkerstma RT, Frederick H, Rattunde W, Chandra S, Soma Raju G, Hash CT: The pattern of genetic diversity of Guinea-race Sorghum bicolor (L.) Moench landraces as revealed with SSR markers. Theor Appl Genet 2005, I I I:399-409.

20. Ritter KB, Mclntyre CL, Godwin ID, Jordan DR, Chapman S: An assessment of the genetic relationships between sweet and grain sorghums, within Sorghum bicolor ssp. bicolor (L.) Moench, using AFLP markers. Euphytica in press.

21. Jordan DR, Tao YZ, Godwin ID, Henzell RG, Cooper M, McIntyre CL: Comparison of identity by descent and identity by state for detecting genetic regions under selection in a sorghum pedigree breeding program. Mol Breed 2004, I 4:44I-454.

22. Yu J, Buckler ES: Genetic association mapping and genome organization in maize. Curr Opin Biotechnol 2006, I7:155-160.

23. Podlich D, Winkler CR, Cooper M: Mapping As You Go: An Effective Approach for Marker-Assisted Selection of Complex Traits. Crop Sci 2004, 44: I560-I57I.

24. Furman BJ: Methodology to establish a composite collection: Case study in lentil. Genet Resour Crop Evol 2006, 4:2-12.

25. Jaccoud D, Peng K, Feinstein D, Kilian A: Diversity arrays: a solid state technology for sequence information independent genotyping. Nucleic Acids Res 200I, 29(4):e25.

26. Wenzl P, Li H, carling J, Zhou M, Raman H, Paul E, Hearnden P, Maier C, Xia L, Caig V, Jaroslava O, Cakir M, Poulsen D, Wang J, Raman R, Smith KP, Muehlbauer GJ, Chalmers KJ, Kleinhofs A, Huttner E, Kilian A: A high-density consensus map of barley linking DArT markers to SSR, RFLP and STS loci and agricultural traits. BMC Genomics 2006, 7:206-228. 
27. Xia L, Peng K, Yang S, Wenzl P, Carmen de Vicente M, Fregene M, Kilian A: DArT for high-throughput genotyping of Cassava (Manihot esculenta) and its wild relatives. Theor Appl Genet 2005, I I 0: 1092-1098

28. Wittenberg AHJ, van der Lee T, Cayla C, Kilian A, Visser RGF, Schouten HJ: Validation of the high-throughput marker technology DArT using the model plant Arabidopsis thaliana. Mol Gen Genomics 2005, 274:30-39.

29. Yang S, Pang W, Ash G, Harper J, Carling J, Wenzl P, Huttner E, Zong $X$, Kilian A: Low level of genetic diversity in cultivated pigeonpea compared to its wild relatives is revealed by diversity arrays technology. Theor Appl Genet 2006, I I 3:585-595.

30. Akbari M, Wenzl P, Caig V, Carling J, Xia L, Yang S, Uszynski G, Mohler V, Lehmensiek A, Kuchel H, Hayden MJ, Howes N, Sharp P Vaughan $P$, Rathnell B, Huttner E, Kilian A: Diversity arrays technology (DArT) for high-throughput profiling of the hexaploid wheat genome. Theor Appl Genet 2006, I I 3: I 409- I 420

31. The Official Site of Diversity Arrays Technology (DArT P/L) [http://www.diversityarrays.com]

32. Kilian A, Huttner E, Wenzl P, Jaccoud D, Carling J, Caig V, Evers M Heller-Uszynska K, Cayla C, Patarapuwadol S, Xia L, Yang S, Thomson $B$ : The fast and the cheap: SNP and DArT-based whole genome profiling for crop improvement. In Proceedings of the International Congress In the Wake of the Double Helix: From the Green Revolution to the Gene Revolution May 27-3I, 2003 Bologna, Italy Edited by: Tuberosa R, Phillips RL, Gale M. Avenue Media; 2005:443-46I.

33. Diatchenko L, Lau YF, Campbell AP, Chenchik A, Moqadam F, Huang B, Lukyanov S, Lukyanov K, Gurskaya N, Sverdlov ED, Siebert PD: Suppression subtractive hybridization: a method for generating differentially regulated or tissue-specific cDNA probes and libraries. Proc Natl Acad Sci USA 1996, 93:6025-30.

34. Parh DK: DNA-based markers for ergot resistance in sorghum. In PhD thesis University of Queensland, School of Land and Food Sciences; 2005.

35. Dillmann C, Bar-Hen A, Guerin D, Charcosset A, Murigneux A: Comparison of RFLP and morphological distances between maize Zea mays L. inbred lines. Consequence for germplasm protection purposes. Theor Appl Genet 1997, 95:92-I02.

36. Altshuler D, Pollar VJ, Cowles CR, Van Etten WJ, Baldwin J, Linton L, Lander ES: A SNP map of the human genome generated by reduced representation shotgun sequencing. Nature 2000, 407:5I3-5 I6.

37. Irizarry K, Kustanovich V, Li C, Brown N, Nelson S, Wong W, Lee C] Genome-wide analysis of single nucleotide polymorphisms in human expressed sequences. Nat Genet 2000, 26:233-236.

38. Wakeley J, Nielsen R, Liu-Cordero SN, Ardlie K: The discovery of single-nucleotide polymorphisms and inferences about human demographic history. Am J Hum Genet 200I, 69:1332-1347

39. Nielsen R: Estimation of population parameters and recombination rates from single nucleotide polymorphisms. Genetics 2000, I 54:931-942.

40. Akey JM, Zhang K, Xiong M, Jin L: The effect of single nucleotide polymorphism identification strategies on estimates of linkage disequilibrium. Mol Biol Evol 2003, 20:232-242.

41. Menz MA, Klein RR, Mullet JE, Obert JA, Unruh NC, Klein PE: A high-density genetic map of Sorghum bicolor (L.) Moench based on 2926 AFLP ${ }^{\circledR}$, RFLP and SSR markers. Plant Mol Biol 2002, 48:483-499.

42. Bowers JE, Abbey C, Anderson S, Chang C, Draye X, Hoppe AH, Jessup R, Lemke C, Lennington J, Li Z, Lin Y, Liu S, Luo L, Marler BS, Ming R, Mitchell SE, Qiang D, Reischmann K, Schulze SR, Skinner DN, Wang Y, Kresovich S, Schertz KF, Paterson AH: A high-density genetic recombination map of sequence-tagged sites for Sorghum, as a framework for comparative structural and evolutionary genomics of tropical grains and grasses. Genetics 2003, 1 65:367-386.

43. Kim J-S, Islam-Faridi MN, Klein PE, Stelly DM, Price HJ, Klein RR, Mullet JE: Comprehensive molecular cytogenetic analysis of sorghum genome architecture: distribution of euchromatin, heterochromatin. Genes and recombination in comparison to rice. Genetics 2005, I7 I: 1963-1976.

44. Saghai-Maroof MA, Soliman KM, Jorgensen RA, Allard RW: Ribosomal DNA spacer-length polymorphisms in barley: Mendelian inheritance, chromosomal location, and population dynamics. Proc Natl Acad Sci USA 1984, 81:8014-80I8.
45. Doyle J, Doyle JL: A rapid DNA isolation procedure for small quantities of fresh leaf material. Phytochemical Bulletin 1987, I9:II-I5.

46. Perrier X, Jacquemoud-Collet JP: DARwin software. 2006 [http:// darwin.cirad.fr/Darwin].

47. Weir BS: Genetic data analysis. Methods for discrete genetic data Sunderland. Mass., Sinauer Associates Inc; 1990.

48. MultiQTL [http://www.multiqtl.com]

49. Mester D, Ronin YI, Hu Y, Nevo E, Korol A: Constructing large scale genetic maps using evolutionary strategy algorithm. Genetics 2003, 165:2269-2282

50. Kim J-S, Klein P, Klein R, Price H, Mullet J, Stelly D: Chromosome identification and nomenclature of Sorghum bicolor. Genetics 2005, 169:1169-1173.

5I. Kosambi D: The estimation of map distances from recombination values. Ann Eugen 1944, I 2: I72-175.

52. Voorrips RE: MapChart: Software for the graphical presentation of linkage maps and QTLs. J Hered 2002, 93:77-78.

53. GRDC Inc [http://www.grdc.com]
Publish with Biomed Central and every scientist can read your work free of charge

"BioMed Central will be the most significant development for disseminating the results of biomedical research in our lifetime. "

Sir Paul Nurse, Cancer Research UK

Your research papers will be:

- available free of charge to the entire biomedical community

- peer reviewed and published immediately upon acceptance

- cited in PubMed and archived on PubMed Central

- yours - you keep the copyright

Submit your manuscript here:

http://www.biomedcentral.com/info/publishing_adv.asp
BiolMedcentral 